\title{
EXISTENCE AND INFINITELY MANY SOLUTIONS FOR AN ABSTRACT CLASS OF HEMIVARIATIONAL INEQUALITIES
}

\author{
CSABA VARGA
}

Received 1 June 2003 and in revised form 4 January 2005

A general method is given in order to guarantee at least one nontrivial solution, as well as infinitely many radially symmetric solutions, for an abstract class of hemivariational inequalities. This abstract class contains some special cases studied by many authors. We remark that, differently from the classical literature, in the proofs we use the Cerami compactness condition and the principle of symmetric criticality for locally Lipschitz functions.

\section{Introduction}

Let $\left(H,\langle\cdot, \cdot\rangle_{H}\right)$ be a real separable Hilbert space and we suppose that the inclusions $H \hookrightarrow$ $L^{l}\left(\mathbb{R}^{N}\right)$ are continuous with the embedding constants $C(l)$, where $l \in\left[2, p_{0}\right]\left(2<p_{0}<\right.$ $\left.2^{\star}=2 N /(N-2)\right)$. We denote by $\|\cdot\|_{H}$ the norm induced on $H$ by the inner product $\langle\cdot, \cdot\rangle_{H}$ and by $\|\cdot\|_{l}$ the norm of $L^{l}\left(\mathbb{R}^{N}\right)$.

Let $f: \mathbb{R}^{N} \times \mathbb{R} \rightarrow \mathbb{R}$ be a continuous function. Several studies have appeared dealing with the existence and multiplicity of nonzero solutions $u \in H$ of the equation

(E)

$$
\langle u, v\rangle_{H}=\int_{\mathbb{R}^{N}} f(x, u(x)) v(x) d x, \quad \forall v \in H .
$$

Existence and multiplicity results in some special cases of (E) were studied in many papers, see for instance Strauss [20], Bartsch and Willem [3, 4], Bartsch and Wang [2], and in the monographs of Kavian [8], Struwe [21], and Willem [22].

Now, let $f: \mathbb{R}^{N} \times \mathbb{R} \rightarrow \mathbb{R}$ be a measurable function, and consider a real number $2<$ $p<p_{0}$, and we suppose that the function $f$ satisfies the growth condition

$\left(\mathrm{f}_{1}\right)|f(x, s)| \leq c\left(|s|+|s|^{p-1}\right)$ for a.e. $x \in \mathbb{R}^{N}$, for all $s \in \mathbb{R}$,

where $c>0$ is a positive constant. In what follows, we use only that the functions $h_{1}(u)=$ $c|u|$ and $h_{2}(u)=c|u|^{p-1}$ are convex, increasing, and $h_{1}(0)=h_{2}(0)=0$.

Let $F: \mathbb{R}^{N} \times \mathbb{R} \rightarrow \mathbb{R}$ be the function defined by

$$
F(x, u)=\int_{0}^{u} f(x, s) d s, \quad \text { for a.e. } x \in \mathbb{R}^{N}, \forall s \in \mathbb{R} .
$$


For a.e. $x \in \mathbb{R}^{N}$, we have

$$
|F(x, u)-F(x, v)| \leq c_{1}|u-v|\left[h_{1}(|u|)+h_{1}(|v|)+h_{2}(|u|)+h_{2}(|v|)\right]
$$

where $c_{1}$ is a constant which does not depend on $u$ and $v$. Therefore, the function $F(x, \cdot)$ is locally Lipschitz and we can define the (partial) Clarke derivative of it, that is,

$$
F_{2}^{0}(x, u ; w)=\limsup _{y \rightarrow u, t \rightarrow 0^{+}} \frac{F(x, y+t w)-F(x, y)}{t}
$$

for a.e. $x \in \mathbb{R}^{N}$ and for all $u, w \in \mathbb{R}$.

Now we formulate the following hemivariational inequality problem.

Problem 1.1. Find $u \in H$ such that

$(\mathrm{P})$

$$
\langle u, v\rangle_{H}+\int_{\mathbb{R}^{N}} F_{2}^{0}(x, u(x) ;-v(x)) \geq 0, \quad \forall v \in H .
$$

To study the existence of solutions of problem $(\mathrm{P})$, we introduce the functional $\Psi$ : $H \rightarrow \mathbb{R}$ defined by $\Psi(u)=(1 / 2)\|u\|_{H}^{2}-\Phi(u)$, where $\Phi(u)=\int_{\mathbb{R}^{N}} F(x, u(x)) d x$. We will see that the critical points (in the sense of Chang) of the functional $\Psi$ are the solutions of problem (P). Therefore, it is enough to study the existence of the critical points of the functional $\Psi$.

Such problems appear in the nonsmooth mechanics, see the books of Panagiotopoulos $[17,18]$, Motreanu and Panagiotopoulos [14]. The study of problem (P) is motivated by these books and by the aforementioned papers. We emphasize that problem $(\mathrm{P})$ was studied in some special cases by Gazzola and Rădulescu [6], and Kristály [11], where the nonsmooth Palais-Smale condition was used. In this paper, we extend the above results to a general case and we use the so-called nonsmooth Cerami compactness condition. More precisely, we say that $\Psi$ satisfies the Cerami (PS) condition at level $c \in \mathbb{R}\left((\mathrm{CPS})_{c}\right)$ if every sequence $\left(u_{n}\right) \subset H$, such that $\Psi\left(u_{n}\right) \rightarrow c$ and $\left(1+\left\|u_{n}\right\|_{H}\right) \lambda_{\Psi}\left(u_{n}\right) \rightarrow 0$, contains a convergent subsequence in $H$ (see [9] or [12]).

We suppose that the function $f: \mathbb{R}^{N} \times \mathbb{R} \rightarrow \mathbb{R}$ satisfies the following condition.

$\left(f_{2}\right)$ There exist $\alpha \in\left(\max \left\{2, p_{0}\left((p-2) /\left(p_{0}-2\right)\right)\right\}, p_{0}\right)$ and a constant $C>0$ such that for a.e. $x \in \mathbb{R}^{N}$ and all $u \in \mathbb{R}$, we have

$$
-C|u|^{\alpha} \geq F(x, u)+\frac{1}{2} F_{2}^{0}(x, u ;-u) .
$$

To study the Cerami compactness condition, we impose further assumptions on $f$. First, we define two functions by

$$
\begin{aligned}
& \underline{f}(x, s)=\lim _{\delta \rightarrow 0^{+}} \operatorname{essinf}\{f(x, t):|t-s|<\delta\}, \\
& \bar{f}(x, s)=\lim _{\delta \rightarrow 0^{+}} \operatorname{esssup}\{f(x, t):|t-s|<\delta\},
\end{aligned}
$$


for every $s \in \mathbb{R}$ and a.e. $x \in \mathbb{R}^{N}$. It is clear that $\underline{f}(x, \cdot)$ is lower semicontinuous and $\bar{f}(x, \cdot)$ is upper semicontinuous. The following hypotheses for $f$ was introduced by Chang [5].

$\left(\mathrm{f}_{3}\right)$ The functions $f, \bar{f}$ are $N$-measurable, that is, for every measurable function $u$ : $\mathbb{R}^{N} \rightarrow \mathbb{R}$, the functions $x \mapsto f(x, u(x)), x \mapsto \bar{f}(x, u(x))$ are measurable.

$\left(\mathrm{f}_{4}\right)$ For every $\varepsilon>0$, there exists $\bar{c}_{\mathcal{\varepsilon}}>0$, such that for a.e. $x \in \mathbb{R}^{N}$ and for every $s \in \mathbb{R}$, we have

$$
|f(x, s)| \leq \varepsilon h_{1}(s)+c(\varepsilon) h_{2}(s),
$$

where the inclusion $H \hookrightarrow L^{2}\left(\mathbb{R}^{N}\right)$ is continuous and the inclusion $H \hookrightarrow L^{p}\left(\mathbb{R}^{N}\right)$ is compact.

Remark 1.2. We observe that, if we impose the following condition on $f$ :

$\left(\mathrm{f}_{4}^{\prime}\right)$ if the inclusion $H \hookrightarrow L^{2}\left(\mathbb{R}^{N}\right)$ is continuous, $H \hookrightarrow L^{p}\left(\mathbb{R}^{N}\right)$ is compact and

$$
\lim _{\varepsilon \rightarrow 0^{+}} \operatorname{esssup}\left\{\left|\frac{f(x, s)}{s}\right|:(x, s) \in \mathbb{R}^{N} \times\right]-\varepsilon, \varepsilon[\}=0,
$$

then this condition with $\left(\mathrm{f}_{1}\right)$ implies $\left(\mathrm{f}_{4}\right)$.

We have the following result.

THEOREM 1.3. If the conditions $\left(f_{1}\right)-\left(f_{4}\right)$ are fulfilled, then $\Psi$ satisfies the $(C P S)_{c}$ condition for every $c>0$.

To prove the existence and multiplicity results for problem $(\mathrm{P})$, we impose that the function $f$ satisfies the following conditions.

$\left(\mathrm{f}_{5}\right)$ For a constant $c^{\star}>0$ and for $\alpha$ from condition $\left(\mathrm{f}_{2}\right)$, we have

$$
F(x, u) \geq c^{\star}\left(|u|^{\alpha}-|u|^{2}\right)
$$

for a.e. $x \in \mathbb{R}^{N}$ and all $s \in \mathbb{R}$.

$\left(\mathrm{f}_{6}\right)$ For a.e. $x \in \mathbb{R}^{N}$ and all $s \in \mathbb{R}, f(x,-s)=-f(x, s)$.

Our main results are the following.

THEOREM 1.4. If the conditions $\left(f_{1}\right)-\left(f_{5}\right)$ are fulfilled, then problem $(P)$ has a nontrivial solution.

THEOREM 1.5. If the conditions $\left(f_{1}\right)-\left(f_{6}\right)$ are fulfilled, then problem $(P)$ has infinitely many distinct solutions.

In what follows, let $G$ be the compact topological group $O(N)$ or a subgroup of $O(N)$. We consider that $G$ acts continuously and linearly isometrically on the Hilbert space $H$. Denote by

$$
H^{G}=\{u \in H \mid g x=x \forall g \in G\}
$$


the fixed point set of the action $G$ on $H$. It is well know that $H^{G}$ is a closed subspace of $H$. We suppose in addition that the next condition is fulfilled.

$\left(f_{6}^{\prime}\right)$ For a.e. $x \in \mathbb{R}^{N}$ and for every $g \in G, s \in \mathbb{R}$, we have $f(g x, s)=f(x, s)$.

If we use the principle of symmetric criticality for locally Lipschitz functions, from the above two theorems, we obtain the following corollaries.

Corollary 1.6. If the conditions $\left(f_{1}\right)-\left(f_{5}\right)$ and $\left(f_{6}^{\prime}\right)$ are fulfilled, then problem $(P)$ has a nontrivial, radially symmetric solution.

Corollary 1.7. If the conditions $\left(f_{1}\right)-\left(f_{6}\right)$ and $\left(f_{6}^{\prime}\right)$ are fulfilled, then problem $(P)$ has infinitely many distinct, radially symmetric solutions.

The above results generalize many classical results to nonsmooth case, and in place of the Palais-Smale condition, the Cerami compactness condition is used.

The paper is organized as follows: in Section 2, some facts about locally Lipschitz functions are given; in Section 3, a key inequality is proved; in Section 4, the nonsmooth Cerami condition is verified for the function $\Psi$; in Section 5, we discuss the mountain pass geometry of the function $\Psi$ and there is a multiplicity result given, while in the last section, several applications are presented.

\section{Basic notions}

Let $\left(H,\langle\cdot, \cdot\rangle_{H}\right)$ be a separable Hilbert space and $H^{\star}$ its topological dual. Let $U \subset H$ be an open set. A function $\Psi: U \rightarrow \mathbb{R}$ is called a locally Lipschitz function if each point $u \in U$ possesses a neighborhood $N_{u}$ of $u$ and a constant $K>0$ which depends on $N_{u}$ such that

$$
\left|f\left(u_{1}\right)-f\left(u_{2}\right)\right| \leq K|| u_{1}-u_{2} \|_{H}, \quad \forall u_{1}, u_{2} \in N_{u} .
$$

The generalized directional derivative of a locally Lipschitz function $\Psi: H \rightarrow \mathbb{R}$ in $u \in$ $U$ in the direction $v \in H$ is defined by

$$
\Psi^{0}(u ; v)=\limsup _{\substack{w \rightarrow u \\ t \backslash 0}} \frac{1}{t}(\Psi(w+t v)-\Psi(w)) .
$$

It is easy to verify that $\Psi^{0}(u ;-v)=(-\Psi)^{0}(u ; v)$ for every $u \in U$ and $v \in H$.

The generalized gradient of $\Psi$ in $u \in H$ is defined as being the subset of $H^{\star}$,

$$
\partial \Psi(u)=\left\{z \in H^{\star}:\langle z, v\rangle \leq \Psi^{0}(u ; v), \forall v \in H\right\}
$$

where $\langle\cdot, \cdot\rangle$ is the duality pairing between $H^{\star}$ and $H$. The subset $\partial \Psi(u) \subset H^{\star}$ is nonempty, convex, $w^{\star}$-compact, and we have

$$
\Psi^{0}(u ; v)=\max \{\langle z, v\rangle: z \in \partial \Psi(u)\}, \quad \forall v \in H .
$$


If $\Psi_{1}, \Psi_{2}: U \rightarrow \mathbb{R}$ are two locally Lipschitz functions, then

$$
\left(\Psi_{1}+\Psi_{2}\right)^{0}(u ; v) \leq \Psi_{1}^{0}(u ; v)+\Psi_{2}^{0}(u ; v)
$$

for every $u \in U$ and $v \in X$. We define the function $\lambda_{\Psi}(u)=\inf \left\{\left\|x^{\star}\right\|: x^{\star} \in \partial \Psi(u)\right\}$. This function is lower semicontinuous and this infimum is attained because $\partial \Psi(u)$ is $w^{\star}$-compact. A point $u \in H$ is a critical point of $\Psi$, if $\lambda_{\Psi}(u)=0$, which is equivalent to $\Psi^{0}(u ; v) \geq 0$ for every $v \in H$. In what follows, we use the following proposition.

Proposition 2.1. Let $\Psi: H \rightarrow \mathbb{R}$ be a locally Lipschitz and take $u \in H$ and $\mu>0$. Then the next assertions are equivalent:

(a) $\Psi^{0}(u, v)+\mu\|v\| \geq 0$, for all $v \in H$;

(b) $\lambda_{\Psi}(u) \leq \mu$.

In the next sections, the following two results are used. The following result is a generalized version of mountain pass theorem, see [9, Theorem 6].

Proposition 2.2. Let $H$ be a Hilbert space, let $\Psi: H \rightarrow R$ be a locally Lipschitz function with $\Psi(0)=0$. Suppose that there exist $v \in H$ and constants $\rho, \beta>0$ such that

(i) $\Psi(u) \geq \beta$ for all $u \in H$ with $\|u\|=\rho$;

(ii) $\|v\|>\rho$ and $\Psi(v) \leq 0$;

(iii) $\Psi$ satisfies $(C P S)_{c}$ with

$$
c=\inf _{\gamma \in \Gamma} \max _{t \in[0,1]} \Psi(\gamma(t)),
$$

where

$$
\Gamma=\{\gamma \in C([0,1], H): \gamma(0)=0, \gamma(1)=v\}
$$

Then $c \geq \alpha$ and $c \in \mathbb{R}$ is a critical value of $\Psi$.

The fountain theorem remains true for locally Lipschitz functions even in the case if we replace the Palais-Smale condition by $(\mathrm{CPS})_{c}$, see [15, Corollary 3.4] and in the differentiable cases see Bartsch [1] or Rabinowitz [19]. Thus we have the following proposition.

Proposition 2.3. Let $H$ be a Hilbert space, $\left\{e_{j} \mid j \in \mathbb{N}\right\}$ an orthonormal basis of $H$, and set $H_{k}=\operatorname{span}\left\{e_{1}, \ldots, e_{k}\right\}$. Let $\Psi: H \rightarrow \mathbb{R}$ be a locally Lipschitz functional which satisfies the following hypotheses:

(i) $\Psi(u)=\Psi(-u)$, for all $u \in H$,

(ii) for every $k \geq 1$, there exists $R_{k}>0$ such that $\Psi(u) \leq \Psi(0)$ for every $u \in H_{k}$ with $\|u\| \geq R_{k}$,

(iii) there exist $k_{0} \geq 1, b>0$, and $\rho>0$ such that $\Psi(u) \geq b$ for every $u \in H_{k_{0}}^{\perp}$ with $\|u\|=\rho$,

(iv) $\Psi$ satisfies the $(C P S)_{c}$ condition for every $c \in \mathbb{R}_{+}$. Then $\Psi$ possesses an unbounded critical value $\left\{c_{k}\right\}_{k \in \mathbb{N}^{\star}}$.

Let $G$ be a compact topological group which acts linearly isometrically on the Hilbert space $H$, that is, the action $G \times H \rightarrow H$ is continuous and for every $g \in G, g: H \rightarrow H$ is a linear isometry. The action on $H$ induces an action of the same type on the dual space 
$H^{\star}$ defined by $\left(g x^{*}\right)(x)=x^{*}(g x)$, for all $g \in G, x \in H$, and $x^{*} \in H^{\star}$. Since

$$
\left\|g x^{*}\right\|=\sup _{\|x\|=1}\left|\left(g x^{*}\right)(x)\right|=\sup _{\|x\|=1}\left|x^{*}(g x)\right|
$$

the isometry assumption for the action of $G$ implies that

$$
\left\|g x^{*}\right\|=\sup _{\|x\|=1}\left|x^{*}(x)\right|=\left\|x^{*}\right\|, \quad \forall x^{*} \in H^{\star}, g \in G .
$$

We suppose that $\Psi: H \rightarrow \mathbb{R}$ is a locally Lipschitz and $G$-invariant function, that is, $\Psi(g x)=\Psi(x)$ for every $g \in G$ and $x \in H$. From Krawcewicz and Marzantowicz [10], we have the relation

$$
g \partial \Psi(x)=\partial \Psi(g x)=\partial \Psi(x), \quad \text { for every } g \in G, x \in H
$$

Therefore the subset $\partial \Psi(x) \subset H^{\star}$ is $G$-invariant, so the function $\lambda_{\Psi}(x)=\inf _{w \in \partial \Psi(x)}\|w\|$, $x \in H$, is $G$-invariant. The fixed points set of the action $G$, that is, $H^{G}=\{x \in H \mid g x=$ $x \forall g \in G\}$ is a closed linear subspace of $H$.

We conclude this section with the principle of symmetric criticality for locally Lipschitz functions. This result was proved first by Palais [16] for differentiable functions and for locally Lipschitz functions by Krawcewicz and Marzantowicz [10].

Theorem 2.4. Let $\Psi: H \rightarrow \mathbb{R}$ be a G-invariant locally Lipschitz function and $u \in H^{G}$. Then $u \in H^{G}$ is a critical point of $\Psi$ if and only if $u$ is a critical point of $\Psi^{G}=\left.\Psi\right|_{H^{G}}: H^{G} \rightarrow \mathbb{R}$.

\section{Some basic results}

In this section, we prove that the function $\Phi: H \rightarrow \mathbb{R}$ given by

$$
\Phi(u)=\int_{\mathbb{R}^{N}} F(x, u(x)) d x, \quad \forall u \in H
$$

is locally Lipschitz on bounded sets of $H$ and its generalized directional derivative satisfies the inequality

$$
\Phi^{0}(u ; v) \leq \int_{\mathbb{R}^{N}} F_{2}^{0}(x, u(x) ; v(x)) d x, \quad \forall u, v \in H
$$

Remark 3.1. The following two results are true for the growth condition $\left(\mathrm{f}_{1}\right)$. Moreover, it is enough to prove them in the case when the function $f$ satisfies the growth condition $|f(x, s)| \leq h(s)$ for a.e. $x \in \mathbb{R}^{N}$, for all $s \in \mathbb{R}$, where $h$ is monotone increasing, convex, with $h(0)=0$ and satisfies the following implication: if $u \in L^{l}\left(\mathbb{R}^{N}\right)$, then $h(u) \in L^{q}\left(\mathbb{R}^{N}\right)$, with $1 / l+1 / q=1$. In our case, $h=h_{1}$ or $h=h_{2}$. In the general case, we replace $h$ by $h_{1}+h_{2}$. 
Proposition 3.2. The functional $\Phi: H \rightarrow \mathbb{R}$, defined by $\Phi(u)=\int_{\mathbb{R}^{N}} F(x, u(x)) d x$, is locally Lipschitz on bounded sets of $H$.

Proof. For every $u, v \in H$, with $\|u\|,\|v\|<r$, we have

$$
\begin{aligned}
\|\Phi(u)-\Phi(v)\| & \leq \int_{\mathbb{R}^{N}}|F(x, u(x))-F(x, v(x))| d x \\
& \leq c_{1} \int_{\mathbb{R}^{N}}|u(x)-v(x)|[h(u(x))+h(v(x))] \\
& \leq c_{2}\left(\int_{\mathbb{R}^{N}}|u(x)-v(x)|^{p}\right)^{1 / p}\left[\left(\int_{\mathbb{R}^{N}}\left(h(u(x))^{q} d x\right)\right)^{1 / q}\right. \\
& \left.\quad+\left(\int_{\mathbb{R}^{N}}\left(h(v(x))^{q} d x\right)\right)^{1 / q}\right] \\
& \leq c_{2}\|u-v\|_{p}\left[\|h(u)\|_{q}+\|h(v)\|_{q}\right] \\
& \leq C(u, v)\|u-v\|_{H},
\end{aligned}
$$

where we used the Hölder inequality, the subadditivity of the norm $\|\cdot\|_{q}$, and the fact that the inclusion $H \hookrightarrow L^{l}\left(\mathbb{R}^{N}\right)$ is continuous. The constant $C(u, v)$ depends only on $u$ and $v$.

Proposition 3.3. If the condition $\left(f_{1}\right)$ holds, then for every $u, v \in H$,

$$
\Phi^{0}(u ; v) \leq \int_{\mathbb{R}^{N}} F_{2}^{0}(x, u(x) ; v(x)) d x .
$$

Proof. It suffices to prove the proposition for the function $f$, which satisfies only the growth condition from Remark 3.1. We fix the elements $u, v \in H$. The function $F(x, \cdot)$ is locally Lipschitz, therefore is continuous. Thus $F_{2}^{0}(x, u(x) ; v(x))$ can be expressed as the upper limit of $(F(x, y+t v(x))-F(x, y)) / t$, where $t \rightarrow 0^{+}$taking rational values and $y \rightarrow$ $u(x)$ taking values in a countable subset of $\mathbb{R}$. Therefore, the map $x \rightarrow F_{2}^{0}(x, u(x) ; v(x))$ is measurable as the "countable limsup" of measurable functions in $x$. From condition $\left(\mathrm{f}_{1}\right)$, we get that the function $x \rightarrow F_{2}^{0}(x, u(x) ; v(x))$ is from $L^{1}\left(\mathbb{R}^{N}\right)$.

Using the fact that the Hilbert space $H$ is separable, there exist a sequence $w_{n} \in E$ with $\left\|w_{n}-u\right\|_{H} \rightarrow 0$ and a real number sequence $t_{n} \rightarrow 0^{+}$, such that

$$
\Phi^{0}(u, v)=\lim _{n \rightarrow \infty} \frac{\Phi\left(w_{n}+t_{n} v\right)-\Phi\left(w_{n}\right)}{t_{n}} .
$$

Because the inclusion $H \hookrightarrow L^{l}\left(\mathbb{R}^{N}\right)$ is continuous, we get $\left\|w_{n}-u\right\|_{l} \rightarrow 0$. Using Brézis [7, Theorem IV.9(a)], there exists a subsequence of $\left(w_{n}\right)$ denoted in the same way, such that $w_{n}(x) \rightarrow u(x)$, a.e. $x \in \mathbb{R}^{N}$. Now, let $\varphi_{n}: \mathbb{R}^{N} \rightarrow \mathbb{R} \cup\{+\infty\}$ be the function defined by

$$
\begin{aligned}
\varphi_{n}(x)= & -\frac{F\left(x, w_{n}(x)+t_{n} v(x)\right)-F\left(x, w_{n}(x)\right)}{t_{n}} \\
& +c_{1}|v(x)|\left[h\left(\left|w_{n}(x)+t_{n} v(x)\right|\right)+h\left(\left|w_{n}(x)\right|\right)\right] .
\end{aligned}
$$


By the relation (1.3), we see that the functions $\varphi_{n}$ are measurable and nonnegative. If we apply Fatou's lemma, we get

$$
\int_{\mathbb{R}^{N}} \liminf _{n \rightarrow \infty} \varphi_{n}(x) d x \leq \liminf _{n \rightarrow \infty} \int_{\mathbb{R}^{N}} \varphi_{n}(x) d x
$$

This inequality is equivalent to

$$
\int_{\mathbb{R}^{N}} \limsup _{n \rightarrow \infty}\left[-\varphi_{n}(x)\right] d x \geq \limsup _{n \rightarrow \infty} \int_{\mathbb{R}^{N}}\left[-\varphi_{n}(x)\right] d x
$$

For the simplicity in the calculus, we introduce the following notations:

(i) $\varphi_{n}^{1}(x)=\left(F\left(x, w_{n}(x)+t_{n} v(x)\right)-F\left(x, w_{n}(x)\right)\right) / t_{n}$;

(ii) $\varphi_{n}^{2}(x)=c_{1}|v(x)|\left[h\left(w_{n}(x)+t_{n} v(x)\right)+h\left(w_{n}(x)\right)\right]$.

With these notations, we have $\varphi_{n}(x)=-\varphi_{n}^{1}(x)+\varphi_{n}^{2}(x)$.

Now we prove the existence of the limit $b=\lim _{n \rightarrow \infty} \int_{\mathbb{R}^{N}} \varphi_{n}^{2}(x) d x$. Since the inclusion $H \hookrightarrow L^{l}\left(\mathbb{R}^{N}\right)$ is continuous and $\left\|w_{n}-u\right\|_{H} \rightarrow 0$, we get $\left\|w_{n}-u\right\|_{l} \rightarrow 0$. If we use Brézis' [7, Theorem IV.9(b)], there exists a positive function $g \in L^{l}\left(\mathbb{R}^{N}\right)$, such that $\left|w_{n}(x)\right| \leq g(x)$ a.e. $x \in \mathbb{R}^{N}$. Using the fact that the function $h$ is monotone increasing, we get

$$
\left|\varphi_{n}^{2}(x)\right| \leq c_{1}|v(x)|[h(g(x)+|v(x)|)+h(g(x))], \quad \text { a.e. } x \in \mathbb{R}^{N}
$$

Moreover, $\varphi_{n}^{2}(x) \rightarrow 2 c_{1}|v(x)| h(u(x))$, a.e. $x \in \mathbb{R}^{N}$. From the Lebesgue dominated convergence theorem, we have

$$
b=\lim _{n \rightarrow \infty} \int_{\mathbb{R}^{N}} \varphi_{n}^{2}(x) d x=\int_{\mathbb{R}^{N}} 2 c_{1}|v(x)| h(u(x)) d x .
$$

If we denote by $I_{1}=\limsup _{n \rightarrow \infty} \int_{\mathbb{R}^{N}}\left[-\varphi_{n}(x)\right] d x$, then using (3.5) and (3.10), we have

$$
I_{1}=\limsup _{n \rightarrow \infty} \int_{\mathbb{R}^{N}}\left[-\varphi_{n}(x)\right] d x=\Phi^{0}(u ; v)-b .
$$

In what follows, we estimate the expression $I_{2}=\int_{\mathbb{R}^{N}} \lim \sup _{n \rightarrow \infty}\left[-\varphi_{n}(x)\right] d x$. We have the inequality

$$
\int_{\mathbb{R}^{N}} \limsup _{n \rightarrow \infty}\left[\varphi_{n}^{1}(x)\right] d x-\int_{\mathbb{R}^{N}} \lim _{n \rightarrow \infty} \varphi_{n}^{2}(x) d x \geq I_{2}
$$

Since $w_{n}(x) \rightarrow u(x)$, a.e. $x \in \mathbb{R}^{N}$, and $t_{n} \rightarrow 0^{+}$, it follows that

$$
\int_{\mathbb{R}^{N}} \lim _{n \rightarrow \infty} \varphi_{n}^{2}(x) d x=2 c_{1} \int_{\mathbb{R}^{N}}|v(x)| h(|u(x)|) d x .
$$


On the other hand, we have

$$
\begin{aligned}
\int_{\mathbb{R}^{N}} \limsup _{n \rightarrow \infty} \varphi_{n}^{1}(x) d x & \leq \int_{\mathbb{R}^{N}} \limsup _{y \rightarrow u(x), t \rightarrow 0^{+}} \frac{F(x, y+t v(x))-F(x, y)}{t} d x \\
& =\int_{\mathbb{R}^{N}} F_{2}^{0}(x, u(x) ; v(x)) d x .
\end{aligned}
$$

Using the relations (3.8), (3.11), (3.12), and the above estimations, we obtain the desired result.

As we pointed out above, in the general case we replace the function $h$ with the sum $h_{1}+h_{2}$.

\section{The Cerami compactness condition}

Theorem 4.1. Assume that the conditions $\left(f_{1}\right),\left(f_{2}\right),\left(f_{3}\right)$, and $\left(f_{4}\right)$ are fulfilled, then $\Psi$ satisfies the $(C P S)_{c}$ condition for every $c>0$.

Proof. Let $\left(u_{n}\right) \subset H$ be a $(\mathrm{CPS})_{c}$ sequence for the function $\Psi$ and $c>0$, then we have $\Psi\left(u_{n}\right) \rightarrow c$ and $\left(1+\left\|u_{n}\right\|_{H}\right) \lambda_{\Psi}\left(u_{n}\right) \rightarrow 0$. Because $\left(1+\left\|u_{n}\right\|_{H}\right) \lambda_{\Psi}\left(u_{n}\right) \rightarrow 0$, then $\left\|u_{n}\right\|_{H} \lambda_{\Psi}$ $\left(u_{n}\right) \rightarrow 0$. From the definition of $\lambda_{\Psi}\left(u_{n}\right)$, the existence of an element $z_{u_{n}}^{\star} \in \partial \Psi\left(u_{n}\right)$ is obtained, such that $\lambda_{\Psi}\left(u_{n}\right)=\left\|z_{u_{n}}^{\star}\right\|_{H^{\star}}$. For every $v \in H$, we have $\left|z_{u_{n}}^{\star}(v)\right| \leq\left\|z_{u_{n}}^{\star}\right\|_{H^{\star}}\|v\|$, therefore $\left\|z_{u_{n}}^{\star}\right\|_{H^{\star}}\|v\| \geq-z_{u_{n}}^{\star}(v)$. If we take $v=u_{n}$, then $\left\|z_{u_{n}}^{\star}\right\|_{H^{\star}}\left\|u_{n}\right\|_{H} \geq-z_{u_{n}}^{\star}\left(u_{n}\right)$.

Using the properties $\Psi^{0}(u, v)=\max \left\{z^{\star}(v) \mid z^{\star} \in \partial \Psi(u)\right\}$ for every $v \in H$, we have $-z^{\star}(v) \geq-\Psi^{0}(u, v)$ for all $z^{\star} \in \partial \Psi(u)$ and $v \in H$. If we take $u=v=u_{n}$ and $z^{\star}=z_{u_{n}}^{\star}$, we get $-z_{u_{n}}^{\star}\left(u_{n}\right) \geq-\Psi^{0}\left(u_{n}, u_{n}\right)$. Therefore $(1 / 2)\left\|z_{u_{n}}^{\star}\right\|_{H^{\star}}\left\|u_{n}\right\|_{H} \geq-\Psi^{0}\left(u_{n},(1 / 2) u_{n}\right)$. So from the above, we obtain

$$
c+1 \geq c+\frac{1}{2}\left\|z_{u_{n}}^{\star}\right\|_{H^{\star}}\left\|u_{n}\right\|_{H} \geq-z_{u_{n}}^{\star}\left(\frac{1}{2} u_{n}\right) \geq-\Psi^{0}\left(u_{n} ; \frac{1}{2} u_{n}\right)
$$

By the above inequality, the property $\Psi^{0}(u, v) \leq\langle u, v\rangle+\Phi^{0}(u,-v)$, condition $\left(\mathrm{f}_{2}\right)$, and Proposition 3.3, we get

$$
\begin{aligned}
c+1 & \geq \Psi\left(u_{n}\right)-\frac{1}{2} \Psi^{0}\left(u_{n} ; u_{n}\right) \\
& =\frac{1}{2}\left\|u_{n}\right\|_{H}^{2}-\Phi\left(u_{n}\right)-\frac{1}{2}\left(\left\|u_{n}\right\|_{H}^{2}+\Phi^{0}\left(u_{n} ;-u_{n}\right)\right) \\
& \geq-\int_{\mathbb{R}^{N}}\left[F\left(x, u_{n}(x)\right)+\frac{1}{\alpha} F_{2}^{0}\left(x, u_{n}(x) ;-u_{n}(x)\right)\right] d x \\
& \geq C \int_{\mathbb{R}^{N}}\left|u_{n}(x)\right|^{\alpha} d x \\
& \geq C\left\|u_{n}\right\|_{\alpha}^{\alpha} .
\end{aligned}
$$

Therefore, the sequence $\left(u_{n}\right)$ is bounded in $L^{\alpha}\left(\mathbb{R}^{N}\right)$. 
By the condition $\left(f_{4}\right)$, one has that for every $\varepsilon>0$, there exists $c(\varepsilon)>0$, such that for a.e. $x \in \mathbb{R}^{N}$,

$$
F(x, u(x)) \leq \frac{\varepsilon}{2}|u(x)|^{2}+\frac{c(\varepsilon)}{p}|u(x)|^{p} .
$$

After integration, we obtain

$$
\Phi(u) \leq \frac{\varepsilon}{2}\|u\|_{2}^{2}+\frac{c(\varepsilon)}{p}\|u\|_{p}^{p} .
$$

By the expression of $\Psi$ and from the inequality $\|u\|_{2} \leq C(2)\|u\|_{H}$, we get

$$
\begin{aligned}
\left(\frac{1}{2}-\frac{\varepsilon C(2)}{2}\right)\left\|u_{n}\right\|_{H}^{2} & \leq \Psi\left(u_{n}\right)+\frac{c(\varepsilon)}{p}\left\|u_{n}\right\|_{p}^{p} \\
& \leq c+1+\frac{c(\varepsilon)}{p}\left\|u_{n}\right\|_{p}^{p} .
\end{aligned}
$$

Now, we examine the behavior of the sequence $\left(\left\|u_{n}\right\|_{p}^{p}\right)$.

We distinguish three cases:

(a) if $p \in(2, \alpha)$, then for every $u \in L^{2}\left(\mathbb{R}^{N}\right) \cap L^{\alpha}\left(\mathbb{R}^{N}\right)$, we have

$$
\|u\|_{p}^{p} \leq\|u\|_{2}^{2(1-s)}\|u\|_{\alpha}^{s \alpha},
$$

where $p=2(1-s)+\alpha s, s \in(0,1)$,

(b) if $p=\alpha$, we get immediately that the sequence $\left(\left\|u_{n}\right\|_{p}^{p}\right)$ is bounded,

(c) if $p \in\left(\alpha, p_{0}\right)$ and $\alpha>p_{0}\left((p-2) /\left(p_{0}-2\right)\right)$, then for every $u \in L^{\alpha}\left(\mathbb{R}^{N}\right) \cap L^{p_{0}}\left(\mathbb{R}^{N}\right)$, we have

$$
\|u\|_{p}^{p} \leq\|u\|_{\alpha}^{(1-s) \alpha}\|u\|_{p_{0}}^{s p_{0}}
$$

where $p=(1-s) \alpha+s p_{0}, s \in(0,1)$.

If we take $\varepsilon \in(0,1 / C(2))$ and use the above affirmations (a), (b), (c), and (4.5), we obtain that the sequence $\left(u_{n}\right)$ is bounded in $H$. Therefore, $u_{n} \rightarrow u$ weakly in $H$. Because the inclusion $H \hookrightarrow L^{p}\left(\mathbb{R}^{N}\right)$ is compact, we get that $u_{n} \rightarrow u$ strongly in $L^{P}\left(\mathbb{R}^{N}\right)$.

In what follows, we estimate the expressions $I_{n}^{1}=\Psi^{0}\left(u_{n} ; u_{n}-u\right)$ and $I_{n}^{2}=\Psi^{0}(u ; u-$ $\left.u_{n}\right)$. First we estimate the expression $I_{n}^{2}=\Psi^{0}\left(u ; u-u_{n}\right)$. We know that $\Psi^{0}(u ; v) \geq z^{\star}(v)$, for all $v \in H$ and $z^{\star} \in \partial \Psi(u)$. Therefore, for every $z_{u}^{\star} \in \partial \Psi(u)$ fixed, we have $\Psi^{0}(u ; u-$ $\left.u_{n}\right) \geq z_{u}^{\star}\left(u-u_{n}\right)$. Since $u_{n} \rightarrow u$ weakly in $H$, then $\liminf \Psi^{0}\left(u ; u-u_{n}\right) \geq 0$.

Now, we estimate the expression $I_{n}^{1}=\Psi^{0}\left(u_{n} ; u_{n}-u\right)$. From $\lambda_{\Psi}\left(u_{n}\right) \rightarrow 0$ follows the existence of a sequence of real numbers $\mu_{n}>0\left(\mu_{n} \rightarrow 0\right)$, such that $\lambda_{\Psi}\left(u_{n}\right) \leq \mu_{n}$. If we use Proposition 2.1, we get $\Psi^{0}\left(u_{n}, u_{n}-u\right)+\mu_{n}\left\|u_{n}-u\right\|_{H} \geq 0$.

Now, we estimate the expression $I_{n}=\Phi^{0}\left(u_{n} ; u-u_{n}\right)+\Phi\left(u ; u-u_{n}\right)$. From the continuity of the functions $h_{1}(s)=c|s|, h_{2}(s)=c|s|^{p-1}$ and the condition $\left(\mathrm{f}_{4}\right)$, it follows that for every $\varepsilon>0$, there exists a $c(\varepsilon)>0$ such that

$$
\max \{|\underline{f}(x, s)|,|\bar{f}(x, s)|\} \leq \varepsilon\left|h_{1}(s)\right|+c_{\varepsilon} h_{2}(s),
$$


for a.e. $x \in \mathbb{R}^{N}$ and for all $s \in \mathbb{R}$. Using this relation and Proposition 3.3, we have

$$
\begin{aligned}
I_{n}= & \Phi^{0}\left(u_{n} ; u-u_{n}\right)+\Phi\left(u ; u-u_{n}\right) \\
\leq & \int_{\mathbb{R}^{N}}\left[F_{2}^{0}\left(x, u_{n}(x) ; u_{n}(x)-u(x)\right)+F_{2}^{0}\left(x, u(x) ; u(x)-u_{n}(x)\right)\right] d x \\
\leq & \int_{\mathbb{R}^{N}}\left[f\left(x, u_{n}(x)\right)\left(u_{n}(x)-u(x)\right)+\bar{f}(x, u(x))\left(u(x)-u_{n}(x)\right)\right] d x \\
\leq & 2 \varepsilon \int_{\mathbb{R}^{N}}\left[h_{1}(u(x))+h_{1}\left(u_{n}(x)\right)\right]\left|u_{n}(x)-u(x)\right| d x \\
& +2 c(\varepsilon) \int_{\mathbb{R}^{N}}\left[h_{2}(u(x))+h_{2}\left(u_{n}(x)\right)\right]\left|u_{n}(x)-u(x)\right| d x .
\end{aligned}
$$

If we use the Hölder inequality and the fact that the inclusion $H \hookrightarrow L^{2}\left(\mathbb{R}^{N}\right)$ is continuous, we get

$$
\begin{aligned}
I_{n} \leq & 2 \varepsilon C(2)\left\|u_{n}-u\right\|_{H}\left(\left\|h_{1}(u)\right\|_{2}+\left\|h_{1}\left(u_{n}\right)\right\|_{2}\right) \\
& +2 c(\varepsilon)\left\|u_{n}-u\right\|_{p}\left(\left\|h_{2}(u)\right\|_{p^{\prime}}+\left\|h_{2}\left(u_{n}\right)\right\|_{p^{\prime}}\right),
\end{aligned}
$$

where $1 / p+1 / p^{\prime}=1$.

Since $u_{n} \rightarrow u$ strongly in $L^{p}\left(\mathbb{R}^{N}\right)$ and if $\varepsilon \rightarrow 0^{+}$, then $\lim \sup I_{n} \leq 0$ as $n \rightarrow \infty$.

Finally, we use the inequality $\Psi^{0}(u ; v) \leq\langle u, v\rangle+\Phi^{0}(u ;-v)$. If we replace $v$ with $-v$, we get $\Psi^{0}(u,-v) \leq-\langle u, v\rangle+\Phi^{0}(u ; v)$, therefore $\langle u, v\rangle \leq \Phi^{0}(u ; v)-\Psi^{0}(u,-v)$.

If in the above inequality in the place of $u$ and $v$ we put one after the other $u=u_{n}$, $v=u-u_{n}$ and $u=u, v=u_{n}-u$, we get

$$
\begin{gathered}
\left\langle u_{n}, u-u_{n}\right\rangle_{H} \leq \Phi^{0}\left(u_{n}, u-u_{n}\right)-\Psi^{0}\left(u_{n} ; u_{n}-u\right), \\
\left\langle u, u_{n}-u\right\rangle_{H} \leq \Phi^{0}\left(u, u_{n}-u\right)-\Psi^{0}\left(u, u-u_{n}\right) .
\end{gathered}
$$

Adding these relations, we have the key inequality

$$
\begin{aligned}
\left\|u_{n}-u\right\|_{H}^{2} & \leq\left[\Phi^{0}\left(u_{n} ; u-u_{n}\right)+\Phi^{0}\left(u ; u-u_{n}\right)\right]-\Psi^{0}\left(u_{n} ; u_{n}-u\right)-\Psi^{0}\left(u ; u-u_{n}\right) \\
& =I_{n}-I_{n}^{1}-I_{n}^{2}
\end{aligned}
$$

Using the above relation and the estimations of $I_{n}, I_{n}^{1}$ and $I_{n}^{2}$, we get $\left\|u_{n}-u\right\|_{H}^{2} \rightarrow 0$ as $n \rightarrow \infty$.

\section{Existence and multiplicity results}

In this section, we prove Theorems 1.4 and 1.5.

Proof of Theorem 1.4. The conditions $\left(\mathrm{f}_{1}\right)-\left(\mathrm{f}_{4}\right)$ assure that the functional $\Psi(u)=$ $(1 / 2)\|u\|_{H}^{2}-\Phi(u)$ satisfies the (CPS) ${ }_{c}$ condition. We verify only the following geometric hypotheses from Proposition 2.2:

$$
\begin{aligned}
& \exists \beta, \rho>0 \text { such that } \Psi \geq \beta \text { on } B_{\rho}(0)=\left\{u \in H:\|u\|_{H}=\rho\right\} ; \\
& \Psi(0)=0, \quad \exists v \in H, \quad\|v\|_{H}>\rho \text { such that } \Psi(v) \leq 0 .
\end{aligned}
$$


For the proof of relation (5.1), we use the relation $\left(\mathrm{f}_{4}\right)$, that is, $|f(x, s)| \leq \varepsilon|s|+c(\varepsilon)$ $|s|^{p-1}$. Integrating this inequality and using that the inclusions $H \hookrightarrow L^{2} \mathbb{R}^{N}, H \hookrightarrow L^{p}\left(\mathbb{R}^{N}\right)$ are continuous, we get that

$$
\Psi(u) \geq\left(\frac{1-\varepsilon C(2)}{2}\right)\|u\|_{H}^{2}-\frac{1}{p} c_{\varepsilon} C(p)\|u\|_{H}^{p} .
$$

The right member of this inequality is a function $\chi: \mathbb{R}_{+} \rightarrow \mathbb{R}$ of the form $\chi(t)=A t^{2}-B t^{p}$, where $A, B>0$ are some constants. The function $\chi$ attains its global maximum in the point $t_{M}=(2 A / p B)^{1 /(p-2)}$. In our case, $A=(1-\varepsilon C(2)) / 2$ and $B=(1 / p) c_{\varepsilon} C(p)$. If we take $\rho=t_{M}$ and $\left.\left.\beta \in\right] 0, \chi\left(t_{M}\right)\right]$, it is easy to see that the condition (5.1) is fulfilled.

By the condition $\left(f_{5}\right)$, we have $\Psi(u) \leq\left(c^{\star} C(\alpha)+1 / 2\right)\|u\|_{H}^{2}-c^{\star}\|u\|_{\alpha}^{\alpha}$, where $C(\alpha)>0$ is the constant of the continuous inclusion $H \hookrightarrow L^{\alpha}\left(\mathbb{R}^{N}\right)$. If we fix an element $v \in H \backslash\{0\}$ and in place of $u$ we put $t v$, then we have

$$
\Psi(t v) \leq\left(c^{\star} C(\alpha)+\frac{1}{2}\right) t^{2}\|v\|_{H}^{2}-c^{\star} t^{\alpha}\|v\|_{\alpha}^{\alpha} .
$$

From this, we see that if $t$ is large enough, then $\|t v\|_{H}>\rho$ and $\Psi(t v)<0$. So, the condition (5.2) is satisfied.

Proof of Theorem 1.5. We prove that the function $\Psi$ verifies the conditions from Proposition 2.3. By the conditions $\left(\mathrm{f}_{1}\right)-\left(\mathrm{f}_{4}\right)$, we get that the function $\Psi$ satisfies the $(\mathrm{CPS})_{c}$ for every $c>0$. From the assumption $\left(f_{6}\right)$, we get that the function $\Psi$ is even, so the requirement (i) from Proposition 2.3 is true. We choose an orthonormal basis $\left\{e_{j}\right\}_{j \in \mathbb{N}^{\star}}$ of $H$ and set $H_{k}=\operatorname{span}\left\{e_{1}, \ldots, e_{k}\right\}$.

As above, we have $\Psi(v) \leq\left(c^{\star} C(\alpha)+1 / 2\right)\|v\|_{H}^{2}-c^{\star}\|v\|_{\alpha}^{\alpha}$. Thus, we have $\Psi(0)=0$. Using the fact that the inclusion $H \hookrightarrow L^{\alpha}\left(\mathbb{R}^{N}\right)$ is continuous, we have that $\left.\|\cdot\|_{\alpha}\right|_{H_{k}}$ is continuous. Because on a finite-dimensional space the continuous norms are equivalent and since $\alpha>2$, there exists an $R_{k}>0$ large enough such that for every $u \in H$ with $\|u\|_{H} \geq R_{k}$, we have $\Psi(u) \leq \Psi(0)=0$. For every $u \in H_{k}^{\perp}$ and $k \in \mathbb{N}^{\star}$, we consider the real numbers $\beta_{k}=\sup _{u \in H_{k}^{\perp} \backslash\{0\}}\left(\|u\|_{p} /\|u\|_{H}\right)$. As in [3, Lemma 3.3], we get $\beta_{k} \rightarrow 0$, if $k \rightarrow \infty$. As in the proof of relation (5.1), we have

$$
\Psi(u) \geq\left(\frac{1-\varepsilon C(2)}{2}\right)\|u\|_{H}^{2}-\frac{1}{p} c_{\varepsilon}\|u\|_{p}^{p} .
$$

By the definition of the number $\beta_{k}$, we have $\|u\|_{p} \leq \beta_{k}\|u\|_{H}$ and combining this with the above relation, we get

$$
\Psi(u) \geq\left(\frac{1-\varepsilon C(2)}{2}\right)\|u\|_{H}^{2}-\frac{1}{p} c_{\varepsilon} \beta_{k}^{p}\|u\|_{H}^{p} .
$$

If we choose $0<\varepsilon<(1 / C(2))((p-2) / p)$ and $\left.\left.r_{k} \in\right]\left(c_{\varepsilon} \beta_{k}^{p}\right)^{1 /(2-p)}\right]$, then we have

$$
\bar{\Psi}(u) \geq\left(\frac{1-\varepsilon C(2)}{2}-\frac{1}{p}\right) r_{k}^{2},
$$


for every $u \in H_{k}^{\perp}$ with $\|u\|_{H}=r_{k}$. Due to the choice of $\varepsilon$ and since $\beta_{k} \rightarrow 0$, the assumptions of Proposition 2.3 are verified. Therefore there exists a sequence of unbounded critical values of $\Psi$, which completes the proof.

For the proofs of Corollaries 1.6 and 1.7, we observe that the inclusions $H^{G} \hookrightarrow L^{l}\left(\mathbb{R}^{N}\right)$ are continuous for $l \in\left[2, p_{0}\right]$ and $H^{G} \hookrightarrow L^{p}\left(\mathbb{R}^{N}\right)$ is compact. Replacing $\Psi$ with $\left.\Psi\right|_{H^{G}}$ and using the principle of symmetric criticality for locally Lipschitz function, we repeat the proofs of Theorems 1.4 and 1.5.

\section{Applications}

In this section, we apply the above results for some particular hemivariational inequalities which generalize several results studied by many authors.

Application 6.1. Let $f: \mathbb{R}^{N} \times \mathbb{R} \rightarrow \mathbb{R}$ be a measurable function. Suppose that the function $f$ satisfies the conditions $\left(\mathrm{f}_{1}\right)-\left(\mathrm{f}_{5}\right)$.

Let $b: \mathbb{R}^{N} \times \mathbb{R} \rightarrow \mathbb{R}$ be a continuous function. For $b$, we will first assume the following.

$\left(b_{1}\right) b_{0}:=\inf _{x \in \mathbb{R}^{N}} b(x)>0$.

$\left(b_{2}\right)$ For every $M>0$,

$$
m\left(\left\{x \in \mathbb{R}^{N}: b(x) \leq M\right\}\right)<\infty,
$$

where $m$ denotes the Lebesgue measure in $\mathbb{R}^{N}$.

We consider the Hilbert space

$$
H:=\left\{u \in W^{1,2}\left(\mathbb{R}^{N}\right): \int_{\mathbb{R}^{N}}\left(|\nabla u|^{2}+b(x) u^{2}\right) d x<\infty\right\},
$$

with the inner product

$$
\langle u, v\rangle_{H}=\int_{\mathbb{R}^{N}}(\nabla u \nabla v+b(x) u v) d x
$$

In the paper, Bartsch and Wang [2] proved that the inclusion $H \hookrightarrow L^{s}\left(\mathbb{R}^{N}\right)$ is compact for $p \in[2,2 N /(N-2))$. Now we formulate the problem.

Problem 6.2. Find a positive $u \in H$ such that for every $v \in H$, we have $\left(P_{1}\right)$

$$
\int_{\mathbb{R}^{N}}(\nabla u \nabla v+b(x) u v) d x+\int_{\mathbb{R}^{N}} F_{2}^{0}(x, u(x) ;-v(x)) d x \geq 0 .
$$

We have the next result which extends a result from Gazzola and Rădulescu [6] and Bartsch and Wang [2].

Corollary 6.3. If the conditions $\left(f_{1}\right)-\left(f_{5}\right)$ and $\left(b_{1}\right)-\left(b_{2}\right)$ hold, then problem $\left(P_{1}\right)$ has a positive solution. 
102 Hemivariational inequalities

Proof. We replace the function $f$ by $f_{+}: \mathbb{R}^{N} \times \mathbb{R} \rightarrow \mathbb{R}$ defined by

$$
f_{+}(x, u)= \begin{cases}f(x, u) & \text { if } u \geq 0 \\ 0 & \text { if } u<0\end{cases}
$$

and we apply Theorem 1.4.

Application 6.4. Now, we consider $A u:=-\triangle u+|x|^{2} u$ for $u \in D(A)$, where

$$
D(A):=\left\{u \in L^{2}\left(\mathbb{R}^{N}\right): A u \in L^{2}\left(\mathbb{R}^{N}\right)\right\}
$$

Here $|\cdot|$ denotes the Euclidean norm of $\mathbb{R}^{N}$.

In this case, the Hilbert space $H$ is defined by

$$
H:=\left\{u \in L^{2}\left(\mathbb{R}^{N}\right): \int_{\mathbb{R}^{N}}\left(|\nabla u|^{2}+|x|^{2} u^{2}\right) d x<\infty\right\}
$$

with the inner product

$$
\langle u, v\rangle_{H}=\int_{\mathbb{R}^{N}}\left(\nabla u \nabla v+|x|^{2} u v\right) d x
$$

The inclusion $H \hookrightarrow L^{s}\left(\mathbb{R}^{N}\right)$ is compact for $p \in[2,2 N /(N-2))$, see Kavian [8, page 278].

We formulate the next problem.

Problem 6.5. Find a positive $u \in H$ such that for every $v \in H$, we have $\left(P_{2}\right)$

$$
\int_{\mathbb{R}^{N}}\left(\nabla u \nabla v+|x|^{2} u v\right) d x+\int_{\mathbb{R}^{N}} F_{2}^{0}(x, u(x) ;-v(x)) d x \geq 0 .
$$

Corollary 6.6. If the conditions $\left(f_{1}\right)-\left(f_{5}\right)$ hold, then problem $\left(P_{2}\right)$ has a positive solution. Corollary 6.7. If the conditions $\left(f_{1}\right)-\left(f_{6}\right)$ hold, then problems $\left(P_{1}\right)$ and $\left(p_{2}\right)$ have infinitely many distinct positive solutions.

Application 6.8. In this application, we consider the Hilbert space $H$ given by

$$
H:=H^{1}\left(\mathbb{R}^{N}\right)=\left\{u \in L^{2}\left(\mathbb{R}^{N}\right): \nabla u \in L^{2}\left(\mathbb{R}^{N}\right)\right\}
$$

with the inner product

$$
\langle u, v\rangle_{H}=\int_{\mathbb{R}^{N}}(\nabla u \nabla v+u v) d x
$$

We consider $G=O(N)$. The group $G$ acts linearly and orthogonally on $\mathbb{R}^{N}$. 
The action of $G$ on $H$ is defined by $g u(x)=u\left(g^{-1} x\right)$ for all $g \in G$ and $x \in \mathbb{R}^{N}$. The fixed point set of this action is

$$
H^{G}=\left\{u \in H^{1}\left(\mathbb{R}^{N}\right) \mid g u=u\right\}
$$

According to a result of Lions [13], the inclusion $H^{G} \hookrightarrow L^{s}\left(\mathbb{R}^{N}\right)$ is compact for $s \in(2,2 N /$ $(N-2))$.

The proposed problem is the following.

Problem 6.9. Find $u \in H$ such that for every $v \in H$, we have

$\left(P_{3}\right)$

$$
\int_{\mathbb{R}^{N}}(\nabla u \nabla v+u v) d x+\int_{\mathbb{R}^{N}} F_{2}^{0}(x, u(x) ;-v(x)) d x \geq 0 .
$$

Corollary 6.10. If the conditions $\left(f_{1}\right)-\left(f_{6}\right)$ and $\left(f_{6}^{\prime}\right)$ are fulfilled, then problem $\left(P_{3}\right)$ has a sequence of radial solutions $\left(u_{k}\right)_{k \in \mathbb{N}} \subset H$ such that $\Psi\left(u_{k}\right) \rightarrow \infty$ as $k \rightarrow \infty$.

Remark 6.11. The above result generalizes the main result from Bartsch and Willem [4], and extends the main result from Kristály [11], where the Palais-Smale condition is used.

We conclude this section with an example which satisfies the conditions $\left(f_{1}\right)-\left(f_{6}\right)$ and $\left(f_{6}^{\prime}\right)$.

Example 6.12. We consider a sequence $\left(a_{n}\right)_{n \in \mathbb{N}}$ of real numbers with $a_{0}=0$ and $a_{n}>0$ for every $n \geq 1$. We introduce the following notations:

$$
A_{n}:=\sum_{k=0}^{n} a_{k}, \quad A:=\sum_{k=0}^{\infty} a_{k} .
$$

We suppose that the series $A=\sum_{k=0}^{\infty} a_{k}$ is convergent with $A>1$. Let $f: \mathbb{R} \rightarrow \mathbb{R}$ be a function defined by $f(s)=s\left(|s|^{p-2}+A_{n}\right)$ for every $s \in[n, n+1) \cup(-n-1,-n]$, where $n \in \mathbb{N}$ and $p>2$ is a fixed real number. The set of discontinuity of $f$ is $D_{f}=Z \backslash\{0\}$. We affirm that for $N \geq 3$ and $p \in(2,2 N /(N-2))$, the function $f$ satisfies the conditions $\left(\mathrm{f}_{1}\right)-\left(\mathrm{f}_{6}\right)$ and $\left(\mathrm{f}_{6}^{\prime}\right)$.

The conditions $\left(f_{1}\right),\left(f_{3}\right),\left(f_{4}\right),\left(f_{6}\right)$, and $\left(f_{6}^{\prime}\right)$ are trivial. We verify only the conditions $\left(\mathrm{f}_{2}\right)$ and $\left(\mathrm{f}_{5}\right)$.

For $u \in[n, n+1)(n \geq 0)$, we have $F(u)=(1 / p) u^{p}-(1 / 2) A_{n} u^{2}-(1 / 2) \sum_{k=0}^{n} a_{k} k^{2} . \mathrm{Be}-$ cause the function $F$ is continuous, the above equality for $F$ holds for $u=n+1$. The function $f$ is continuous for $u \in(n, n+1)$, therefore $F^{0}(u ;-u)=-u f(u)$. From the definition of $f$, it follows that $F^{0}(u ;-u)$ is left continuous in the discontinuous points of $f$. Therefore, $F^{0}(u ;-u)=-u^{2}\left(|u|^{p-2}+A_{n}\right)$ for every $u \in(n, n+1]$. If we take $\alpha=p>2$ and $C=1 / 2-1 / p$, we have

$$
F(u)+\frac{1}{2} F^{0}(u ;-u) \leq-C|u|^{p}, \quad \forall u \geq 0 .
$$


Because the functions $F(u)$ and $F^{0}(u ;-u)$ are even, the above inequality holds for every $u \in \mathbb{R}$. Therefore the condition $\left(\mathrm{f}_{2}\right)$ is true. For $\alpha=p$ and $c^{\star}=1 / p$, it is easy to see that the condition $\left(f_{5}\right)$ is satisfied.

\section{Acknowledgment}

This work was partially supported by the Research Center of Sapientia Foundation.

\section{References}

[1] T. Bartsch, Infinitely many solutions of a symmetric Dirichlet problem, Nonlinear Anal. 20 (1993), no. $10,1205-1216$.

[2] T. Bartsch and Z. Q. Wang, Existence and multiplicity results for some superlinear elliptic problems on $\mathbf{R}^{N}$, Comm. Partial Differential Equations 20 (1995), no. 9-10, 1725-1741.

[3] T. Bartsch and M. Willem, Infinitely many nonradial solutions of a Euclidean scalar field equation, J. Funct. Anal. 117 (1993), no. 2, 447-460.

[4] Infinitely many radial solutions of a semilinear elliptic problem on $\mathbf{R}^{N}$, Arch. Rational Mech. Anal. 124 (1993), no. 3, 261-276.

[5] K. C. Chang, Variational methods for nondifferentiable functionals and their applications to partial differential equations, J. Math. Anal. Appl. 80 (1981), no. 1, 102-129.

[6] F. Gazzola and V. Rădulescu, A nonsmooth critical point theory approach to some nonlinear elliptic equations in $\mathbb{R}^{n}$, Differential Integral Equations 13 (2000), no. 1-3, 47-60.

[7] H. Brézis, Analyse Fonctionnelle, Collection Mathématiques Appliquées pour la Maîtrise. [Collection of Applied Mathematics for the Master's Degree], Masson, Paris, 1983.

[8] O. Kavian, Introduction à la théorie des points critiques et applications aux problèmes elliptiques, Mathématiques \& Applications (Berlin), vol. 13, Springer, Paris, 1993.

[9] N. C. Kourogenis and N. S. Papageorgiou, Nonsmooth critical point theory and nonlinear elliptic equations at resonance, Kodai Math. J. 23 (2000), no. 1, 108-135.

[10] W. Krawcewicz and W. Marzantowicz, Some remarks on the Lusternik-Schnirelman method for nondifferentiable functionals invariant with respect to a finite group action, Rocky Mountain J. Math. 20 (1990), no. 4, 1041-1049.

[11] A. Kristály, Infinitely many radial and non-radial solutions for a class of hemivariational inequalities, to appear in Rocky Mountain J. Math.

[12] A. Kristály, V. V. Motreanu, and Cs. Varga, A minimax principle with a general Palais-Smale condition, Commun. Appl. Anal. 9 (2005), 285-298.

[13] P.-L. Lions, Symétrie et compacité dans les espaces de Sobolev, J. Funct. Anal. 49 (1982), no. 3, 315-334 (French).

[14] D. Motreanu and P. D. Panagiotopoulos, Minimax Theorems and Qualitative Properties of the Solutions of Hemivariational Inequalities, Nonconvex Optimization and Its Applications, vol. 29, Kluwer Academic Publishers, Dordrecht, 1999.

[15] D. Motreanu and Cs. Varga, A nonsmooth equivariant minimax principle, Commun. Appl. Anal. 3 (1999), no. 1, 115-130.

[16] R. S. Palais, The principle of symmetric criticality, Comm. Math. Phys. 69 (1979), no. 1, 19-30.

[17] P. D. Panagiotopoulos, Inequality Problems in Mechanics and Applications Convex and Nonconvex Energy Functions, Birkhäuser, Massachusetts, 1985.

[18] - Hemivariational Inequalities. Applications in Mechanics and Engineering, Springer, Berlin, 1993.

[19] P. H. Rabinowitz, Minimax Methods in Critical Point Theory with Applications to Differential Equations, CBMS Regional Conference Series in Mathematics, vol. 65, American Mathematical Society, Rhode Island, 1986. 
[20] W. A. Strauss, Existence of solitary waves in higher dimensions, Comm. Math. Phys. 55 (1977), no. 2, 149-162.

[21] M. Struwe, Variational Methods Applications to Nonlinear Partial Differential Equations and Hamiltonian Systems, Springer, Berlin, 1990.

[22] M. Willem, Minimax Theorems, Progress in Nonlinear Differential Equations and Their Applications, vol. 24, Birkhäuser, Massachusetts, 1996.

Csaba Varga: Department of Mathematics, Faculty of Mathematics and Computer Science, BabeşBolyai University, 1 Mihail Kogălniceanu Street, 400084 Cluj-Napoca, Romania

E-mail address: csvarga@math.ubbcluj.ro 